\title{
DEFINITE INTEGRAL OF LOGARITHMIC FUNCTIONS AND POWERS IN TERMS OF THE LERCH FUNCTION ${ }^{1}$
}

\author{
Robert Reynolds ${ }^{\dagger}, \quad$ Allan Stauffer ${ }^{\dagger \dagger}$ \\ Department of Mathematics and Statistics, \\ York University, 4700 Keele Street, Toronto, Canada \\ ${ }^{\dagger}$ milver@my.yorku.ca, ${ }^{\dagger \dagger}$ stauffer@yorku.ca
}

Abstract: A family of generalized definite logarithmic integrals given by

$$
\int_{0}^{1} \frac{\left(x^{i m}(\log (a)+i \log (x))^{k}+x^{-i m}(\log (a)-i \log (x))^{k}\right)}{(x+1)^{2}} d x
$$

built over the Lerch function has its analytic properties and special values listed in explicit detail. We use the general method as given in [5] to derive this integral. We then give a number of examples that can be derived from the general integral in terms of well known functions.

Keywords: Entries of Gradshteyn and Ryzhik, Lerch function, Knuth's Series.

\section{Introduction}

In connection with logarithmic integrals, the authors have the opportunity to evaluate integrals of the form

$$
\int_{0}^{1} \frac{\left(x^{i m}(\log (a)+i \log (x))^{k}+x^{-i m}(\log (a)-i \log (x))^{k}\right)}{(x+1)^{2}} d x
$$

in terms of the Lerch function. We chose this integral as it forms the general case for some integrals published in the Tables of Gradshteyn and Rhyzik. It yields some very interesting special cases in terms of Euler-Mascheroni constant $(\gamma)$, and a pair of Zeta function values $\zeta(1 / 2)$ and $\zeta(-1 / 2)$. The constant $\zeta(1 / 2)$ is used to calculate Knuth's Series and a new integral representation for this constant is derived. The Lerch function is also used in the Bose-Einstein condensation for an exponential density of states function [4]. We also provide formal derivations for some definite integrals in [3] not previously listed in current literature along with new definite integrals in terms of special functions. In our case the constants in the equation (1.1) are general complex numbers subject to the restrictions given below. The derivations follow the method used by us in [5]. The generalized Cauchy's integral formula is given by

$$
\frac{y^{k}}{k !}=\frac{1}{2 \pi i} \int_{C} \frac{e^{w y}}{w^{k+1}} d w
$$

This method involves using a form of equation (1.2) then multiply both sides by a function, then takes a definite integral of both sides. This yields a definite integral in terms of a contour integral. Then we multiply both sides of equation (1.2) by another function and take the infinite sum of both sides such that the contour integral of both equations are the same.

\footnotetext{
${ }^{1}$ This research is supported by NSERC Canada under Grant 504070. The authors confirm there are no conflicts of interest.
} 


\section{Definite integral of the contour integral}

We use the method given in [5]. The contour integral is over $\alpha=m+w$. Here the contour is in the upper left quadrant with $\Im(\alpha)<0$ and going round the origin with zero radius. Using a generalization of Cauchy's integral formula we first replace $y$ by $\log (a)+i x$ then multiply by $e^{m x i}$ for the first equation and then $y$ by $\log (a)-i x$ and multiply by $e^{-m x i}$ to get the second equation followed by replacing $x$ by $\log (x)$. Then we add these two equations, followed by multiplying both sides by $1 / 2(x+1)^{2}$ to get the equality

$$
\frac{\left(x^{i m}(\log (a)+i \log (x))^{k}+x^{-i m}(\log (a)-i \log (x))^{k}\right)}{2(x+1)^{2} k !}=\frac{1}{2 \pi i} \int_{C} \frac{a^{w} w^{-k-1} \cos (\alpha \log (x))}{(x+1)^{2}} d \alpha .
$$

Next we take the definite integral of equation (2.1) over $x \in[0,1]$ to get the following relations

$$
\begin{gathered}
\int_{0}^{1} \frac{\left(x^{i m}(\log (a)+i \log (x))^{k}+x^{-i m}(\log (a)-i \log (x))^{k}\right)}{2(x+1)^{2} k !} d x \\
=\frac{1}{2 \pi i} \int_{0}^{1} \int_{C} \frac{a^{w} w^{-k-1} \cos (\alpha \log (x))}{(x+1)^{2}} d \alpha d x \\
=\frac{1}{2 \pi i} \int_{C} \int_{0}^{1} \frac{a^{w} w^{-k-1} \cos (\alpha \log (x))}{(x+1)^{2}} d x d \alpha \\
=\frac{1}{2 \pi i} \int_{C} \frac{1}{2} \pi(m+w) a^{w} w^{-k-1} \operatorname{csch}(\pi(m+w)) d w
\end{gathered}
$$

from equation (3.883.1) in [3] where the logarithmic function is defined in equation (4.1.2) in [1]. The integral is valid for $a, k$ and $m$ complex and $\Im(\alpha)<0$.

\section{Infinite sum of the contour integral}

In this section we will again use the generalized Cauchy's integral formula to derive equivalent contour integrals. First we replace $y$ by $\log (a)+\pi(2 y+1))$ and multiply both sides by $-m \pi e^{\pi m(2 y+1)}$ to get

$$
-\frac{\pi^{k+1} m e^{\pi m(2 y+1)}(\log (a) / \pi+2 y+1)^{k}}{k !}=-\frac{1}{2 \pi i} \int_{C} \pi \alpha w^{-k-1} \exp (w \log (a)+\pi \alpha(2 y+1)) d \alpha .
$$

Next we take the infinite sum over $y \in[0, \infty)$ simplify the left-hand in terms of the Lerch function side to get

$$
\begin{gathered}
-\frac{2^{k} \pi^{k+1} e^{\pi m} m}{k !} \Phi\left(e^{2 m \pi},-k, \frac{\log (a)+\pi}{2 \pi}\right) \\
=-\frac{1}{2 \pi i} \sum_{y=0}^{\infty} \int_{C} \pi m w^{-k-1}(\exp (w \log (a)+\pi \alpha(2 y+1))) d \alpha \\
=-\frac{1}{2 \pi i} \int_{C} \sum_{y=0}^{\infty} \pi m w^{-k-1}(\exp (w \log (a)+\pi \alpha(2 y+1))) d \alpha \\
=\frac{1}{2 \pi i} \int_{C} \frac{1}{2} \pi m a^{w} w^{-k-1} \operatorname{csch}(\pi \alpha) d \alpha .
\end{gathered}
$$


Next we derive the second contour integral by replacing $k$ by $k-1$ and dropping the linear factor $m$ in equation (3.1) to get

$$
-\frac{2^{k-1} \pi^{k} e^{\pi m} \Phi\left(e^{2 m \pi}, 1-k,(\log (a)+\pi) / 2 \pi\right)}{(k-1) !}=\frac{1}{2 \pi i} \int_{C} \frac{1}{2} \pi a^{w} w^{-k} \operatorname{csch}(\pi \alpha) d \alpha
$$

from (1.232.3) in [3], where $\operatorname{csch}(\mathrm{x})=i \csc (\mathrm{ix})$ from (4.5.10) in [1] and $\Im(\alpha)<0$ for the sum to converge.

We use (9.550) and (9.556) in [3] where $\Phi(z, s, v)$ is the Lerch function which is a generalization of the Hurwitz Zeta and polylogarithm functions.

The Lerch function has a series representation given by

$$
\Phi(z, s, v)=\sum_{n=0}^{\infty}(v+n)^{-s} z^{n}
$$

where $|z|<1, v \neq 0,-1, .$. and is continued analytically by its integral representation given by

$$
\Phi(z, s, v)=\frac{1}{\Gamma(s)} \int_{0}^{\infty} \frac{t^{s-1} e^{-v t}}{1-z e^{-t}} d t=\frac{1}{\Gamma(s)} \int_{0}^{\infty} \frac{t^{s-1} e^{-(v-1) t}}{e^{t}-z} d t
$$

where $\operatorname{Re}(v)>0$, or $|z| \leq 1, z \neq 1, \operatorname{Re}(s)>0$, or $z=1, \operatorname{Re}(s)>1$.

\section{Definite integral in terms of the Lerch function}

Since the right-hand sides of equation (2.2) and (3.1) are equivalent we can equate the left-hand sides simplifying the factorials to get

$$
\begin{gathered}
\int_{0}^{1} \frac{\left(x^{i m}(\log (a)+i \log (x))^{k}+x^{-i m}(\log (a)-i \log (x))^{k}\right)}{(x+1)^{2}} d x \\
=(2 \pi)^{k}\left(-e^{\pi m}\right)\left(k \Phi\left(e^{2 m \pi}, 1-k, \frac{\log (a)+\pi}{2 \pi}\right)+2 \pi m \Phi\left(e^{2 m \pi},-k, \frac{\log (a)+\pi}{2 \pi}\right)\right) .
\end{gathered}
$$

\section{Derivation of entry (4.325.3) in [3]}

In this section will derive an integral representation for the Riemann zeta function. Using equation (4.1) setting $m=0, a=1$ and simplifying the left-hand side we get

$$
\int_{0}^{1} \frac{\log ^{k}(1 / x)}{(x+1)^{2}} d x=2^{-k}\left(2^{k}-2\right) \zeta(k) \Gamma(k+1) .
$$

This formula is equivalent to applying integration by parts to equation (1.12.5) in [2].

Next we take the partial derivative with respect to $k$ of equation (5.1) simplifying to get

$$
\begin{gathered}
\int_{0}^{1} \frac{\log (\log (1 / x)) \log ^{k}(1 / x)}{(x+1)^{2}} d x \\
=2^{-k} \Gamma(k+1)\left(\left(2^{k}-2\right) \zeta^{\prime}(k)+\zeta(k)\left(\left(2^{k}-2\right) \psi^{(0)}(k+1)+\log (4)\right)\right) .
\end{gathered}
$$

Next we set $k=0$ and simplify to get

$$
\int_{0}^{1} \log (\log (1 / x)) \frac{d x}{(x+1)^{2}}=\frac{1}{2}(\log (\pi / 2)-\gamma)
$$

from [7, p. 236]. 


\section{Derivation of special case in terms of $\zeta(-1 / 2)$}

Using equation (5.2) and setting $k=-1 / 2$ we get

$$
\begin{gathered}
\int_{0}^{1} \frac{\log (\log (1 / x))}{\sqrt{\log (1 / x)}} \frac{d x}{(x+1)^{2}} \\
=\sqrt{\pi}\left(2 \sqrt{2} \zeta(-1 / 2) \log (2)-(2 \sqrt{2}-1)\left(\zeta^{\prime}(-1 / 2)+\zeta(-1 / 2) \psi^{(0)}(1 / 2)\right)\right) .
\end{gathered}
$$

\section{Derivation of special cases in terms of $\zeta(1 / 2)$}

Using equation (5.2) and setting $k=1 / 2$ we get

$$
\begin{gathered}
\int_{0}^{1} \sqrt{\log (1 / x)} \log (\log (1 / x)) \frac{d x}{(x+1)^{2}} \\
=\frac{\sqrt{\pi}}{8} \zeta(1 / 2)\left(8-8 \sqrt{2}+2(\sqrt{2}-1) \gamma+\pi+\sqrt{2}\left(\log \left(64 / \pi^{2}\right)-\pi\right)+\log \left(\pi^{2} / 4\right)\right)
\end{gathered}
$$

from [7, p. 236].

\section{Derivation of a special case involving combinations of rational functions of} $\log (x)$ and powers

\subsection{Definite integral in terms of the hypergeometric and Lerch functions}

Setting $k=-1$ and replacing $a$ by $e^{a}$ in (4.1) we derive one equation by replacing $m$ by $i p$ and a second equation by replacing $m$ by $-i p$ then subtracting the two equations and simplifying to get

$$
\begin{gathered}
\int_{0}^{1} \frac{\left(x^{p}-x^{-p}\right) \log (x)}{\left(a^{2}+\log ^{2}(x)\right)} \frac{d x}{(x+1)^{2}} \\
=\frac{1}{4 \pi(a+\pi)}\left(i e^{-i \pi p}(a+\pi) \Phi\left(e^{-2 i p \pi}, 2, \frac{a+\pi}{2 \pi}\right)-i(a+\pi) e^{i \pi p} \Phi\left(e^{2 i p \pi}, 2, \frac{a+\pi}{2 \pi}\right)\right. \\
\left.-4 \pi^{2} p e^{-i \pi p}\left({ }_{2} F_{1}\left(1, \frac{a+\pi}{2 \pi} ; \frac{1}{2}\left(\frac{a}{\pi}+3\right) ; e^{-2 i p \pi}\right)+e_{2}^{i \pi p} F_{1}\left(1, \frac{a+\pi}{2 \pi} ; \frac{1}{2}\left(\frac{a}{\pi}+3\right) ; e^{2 i p \pi}\right)\right)\right)
\end{gathered}
$$

from equation (9.559) in [3] and where $\Re(a)>0$. This is a new entry for Table 4.282 in [3].

\subsection{Definite integral in terms of the Lerch functions}

Setting $k=-2$ and replacing $a$ by $e^{a}$ in (4.1) we derive one equation by replacing $m$ by $i p$ and a second equation by replacing $m$ by $-i p$ then subtracting the two equations and simplifying to get

$$
\begin{gathered}
\int_{0}^{1} \frac{\left(x^{p}-x^{-p}\right) \log (x)}{\left(a^{2}+\log ^{2}(x)\right)^{2}} \frac{d x}{(x+1)^{2}}=\frac{1}{8 \pi^{2} a}\left(-\pi p e^{-i \pi p} \Phi\left(e^{-2 i p \pi}, 2, \frac{a+\pi}{2 \pi}\right)\right. \\
\left.+i e^{-i \pi p} \Phi\left(e^{-2 i p \pi}, 3, \frac{a+\pi}{2 \pi}\right)-e^{i \pi p}\left(\pi p \Phi\left(e^{2 i p \pi}, 2, \frac{a+\pi}{2 \pi}\right)+i \Phi\left(e^{2 i p \pi}, 3, \frac{a+\pi}{2 \pi}\right)\right)\right),
\end{gathered}
$$

where $\Re(a)>0$. This is a new entry for Table 4.282 in [3]. 


\section{Derivation of a special case of combinations involving powers of the logarithm and other powers}

\subsection{Derivation in terms of the hyperbolic tangent and Lerch functions}

Setting $k=-1$ and $a=1$ in (4.1) we derive one equation by replacing $m$ by $i p$ and a second equation by replacing $m$ by $-i p$ then subtracting the two equations and simplifying to get

$$
\int_{0}^{1} \frac{\left(x^{p}-x^{-p}\right)}{(x+1)^{2}} \frac{d x}{\log (x)}=-p \tanh ^{-1}(\cos (\pi p))+\frac{i}{4 \pi}\left(e^{-i \pi p} \Phi\left(e^{-2 i p \pi}, 2, \frac{1}{2}\right)-e^{i \pi p} \Phi\left(e^{2 i p \pi}, 2, \frac{1}{2}\right)\right)
$$

from equations (9.121.27) and (9.559) in [3]. This is a new entry for Table 4.283 in [3].

\subsection{Derivation in terms of Lerch function}

Setting $a=1$ in (4.1) we derive one equation by replacing $m$ by $i p$ and a second equation by replacing $m$ by -ip then subtracting the two equations and simplifying the logarithmic functions on the left-hand side to get

$$
\begin{aligned}
& \int_{0}^{1} \log ^{k}(1 / x)\left(x^{p}-x^{-p}\right)(x+1)^{2} d x=i 2^{k-1} \pi^{k} \csc \left(\frac{\pi k}{2}\right)\left(-k e^{-i \pi p} \Phi\left(e^{-2 i p \pi}, 1-k, \frac{1}{2}\right)\right. \\
& \left.+2 i \pi p e^{-i \pi p} \Phi\left(e^{-2 i p \pi},-k, \frac{1}{2}\right)+e^{i \pi p}\left(k \Phi\left(e^{2 i p \pi}, 1-k, \frac{1}{2}\right)+2 i \pi p \Phi\left(e^{2 i p \pi},-k, \frac{1}{2}\right)\right)\right) .
\end{aligned}
$$

This is a new entry for Table 4.272 in [3].

\section{Discussion}

In this paper we have derived a new integral representation for $\zeta(1 / 2)$ the value of which is apparently unknown in terms of known constants. We were able to derive an efficient method for evaluating Knuth's series using this integral representation. We also derived a new integral representation for evaluating $\zeta(-1 / 2)$. We have dealt with a similar integral in the paper "A Definite Integral Involving the Logarithmic Function in Terms of the Lerch Function" [6]. The present paper should be seen as an extension of these results.

\section{Conclusion}

In this paper, we have presented a novel method for deriving some interesting definite integrals using contour integration. The results presented were numerically verified for both real and imaginary and complex values of the parameters in the integrals using Mathematica by Wolfram.

\section{REFERENCES}

1. Abramowitz M., Stegun I. A. (Eds.) Handbook of Mathematical Functions: with Formulas, Graphs, and Mathematical Tables, 9th printing. New York, Dover, 1972. 1046 p.

2. Erdélyi A., Magnus W., Oberhettinger F., Tricomi F. G. Higher Transcendental Functions. Vol. 1. New York-Toronto-London: McGraw-Hill Book Company Inc., 1953. 316 p.

3. Gradshteyn I. S., Ryzhik I. M. Table of Integrals, Series and Products, 7 ed. Academic Press, 2007. $1171 \mathrm{p}$.

4. Momeni D. Bose-Einstein condensation for an exponential density of states function and Lerch zeta function. Phys. A, 2020. Vol. 541, Art. No. 123264.9 p. DOI: 10.1016/j.physa.2019.123264 
5. Reynolds R., Stauffer A. A method for evaluating definite integrals in terms of special functions with examples. Int. Math. Forum, 2020. Vol. 15, No. 5. P. 235-244. DOI: 10.12988/imf.2020.91272

6. Reynolds R., Stauffer A. A Definite Integral Involving the Logarithmic Function in Terms of the Lerch Function. Mathematics, 2019. Vol. 7, No. 12. Art. No. 1148. 5 p. DOI: $10.3390 /$ math7121148

7. Whittaker E. T., Watson G. N. A Course of Modern Analysis, 4th ed. Cambridge, England: Cambridge University Press, 1996. 608 p. 